\title{
Editorial: 5G-and-Beyond Communications for Smart Cities: Networks, Systems and Applications
}

\author{
Minghua Xia ${ }^{1 *}$, Edmund Lai ${ }^{2}$, Jie Hu ${ }^{3}$, Xuan Liu ${ }^{4}$, Nikolaos Thomos ${ }^{5}$, Zilong Liu ${ }^{5}$, Tao Han ${ }^{6}$, \\ Vivek A. Bohara ${ }^{7}$ and Aduwati Sali ${ }^{8}$ \\ ${ }^{1}$ School of Electronics and Information Technology, Sun Yat-sen University, Guangzhou, China, ${ }^{2}$ School of Engineering, \\ Computer and Mathematical Sciences, Auckland University of Technology, Auckland, New Zealand, ${ }^{3}$ School of Information and \\ Communication Engineering, University of Electronic Science and Technology of China, Chengdu, China, ${ }^{4}$ College of Information \\ Engineering (College of Artificial Intelligence), Yangzhou University, Yangzhou, China, ${ }^{5}$ School of Computer Science and Electronic \\ Engineering, University of Essex, Colchester, United Kingdom, ${ }^{6}$ Department of Electrical and Computer Engineering, New Jersey \\ Institute of Technology, Newark, NJ, United States, ${ }^{7}$ Department of Electronics and Communication Engineering, Institute of \\ Information Technology (IIIT-Delhi), New Delhi, India, ${ }^{8}$ Department of Computer and Communication Systems Engineering, Putra \\ Malaysia University, Selangor Darul Ehsan, Malaysia
}

Keywords: 5G-and-beyond, smart cities (SC), reconfigurable intelligent surface (RIS), urban air mobility (UAM), smart grid, NOMA (non - orthogonal multiple access), prospect theory

Editorial on the Research Topic

5G-and-Beyond Communications for Smart Cities: Networks, Systems and Applications

We are delighted to introduce the readers to the Wireless Communications section of Frontiers in Communications and Networks Journal. It aims to publish high-quality fundamental and applied research in the general area of wireless communications, which play a key role in modern science and engineering. Furthermore, 5G-and-Beyond Communications for Smart Cities is one of the most popular topics. This special issue targets a timely coverage of the latest research advances on cuttingedge communication and networking techniques which have played an increasingly instrumental role in smart cities. Specifically, we aim to investigate and address the following two major research problems: 1) How 5G-and-beyond communication and networking technologies are tailored and optimized for efficiently and intelligently serving different vertical industries (such as transportation, energy, water supply, manufacturing, and telemedicine) in modern cities, and 2) How different AI and ML techniques can work along with these information and communications technologies for enhanced delivery of a vast range of new services and businesses. The research outcomes of this special issue are expected to influence both academia and industry working on smart cities, as well as to generate a significant impact on relevant policymakers and service providers for the employment of new technologies. This research topic focuses on the state-of-the-art of 5G-and-beyond communications, networks, and systems, as well as their applications/services in smart cities. The themes of interest include, but are not limited to:

- 5G-and-beyond network architecture;

- 5G-and-beyond access and transmission technologies;

- 5G network function virtualization/network slicing/orchestration;

- $\mathrm{AI} /$ machine learning-enabled optimization and communications for smart cities;

- Cloud/edge/fog/dew computing and applications/services for smart cities;

- Cyber-physical systems;

- Internet of Things/Everything (IoT/IoE);

- IoT applications/services with ultra-low latency and/or enhanced security; 
- IoT-enabled smart grid;

- Machine-type communications and non-orthogonal multiple access (NOMA) communications for massive connectivity;

- Smart factory/intelligent manufacturing-Industry 4.0;

- Smart home such as smart metering and remote health care monitoring;

- Smart mobilities including intelligent transportation, UAVbased communications, cellular V2X, dedicated short-range communications (DSRC), and visible light communications (VLC);

- Video surveillance and city securities.

It is anticipated that new research on these themes can lead to new design paradigms and solutions for safer, faster, greener, and smarter cities. We received a total of seven submissions, and after a rigorous review process, five articles have been selected for publication, which is each briefly discussed next.

The first article is entitled "Prospect Theory for HumanCentric Communications", by Luo et al. introduces the prospect theory as a promising methodology for modeling user experience and perceptual measurements for humancentric communications. As the founding pillar of behavioral economics, the prospect theory proposes the non-linear quantity and probability perception of human psychology, which extends to five fundamental behavioral attributes that have profound implications for diverse disciplines. Moreover, an example of applying the novel theoretic framework is also provided to illustrate how the prospect theory can be utilized to incorporate human factors and analyze human-centric communications. By expatiating on the prospect theoretic framework, Luo et al. aim to provide a guideline for developing human-centric communications and articulate a novel interdisciplinary research area for further investigation.

The second article is entitled "Urban Air Mobility-A 6G Use Case?", authored by Ansari et al.. Given the successes of unmanned aerial vehicles (UAVs), researchers are already gearing towards aerial transport systems that consist of dense deployment of both UAVs and Personal Aerial Vehicles (PAVs) with human passengers. Although the network key performance indicators of the fifth-generation mobile networks have been optimized to support drone use cases for both high data rates and low latency applications, future aerial transport systems will require stricter network key performance indicators to support the expected massive deployment of aerial vehicles accounting for network capacity and distance between the base stations and the aerial vehicles, among others. Therefore, Ansari et al. present perspective, vision, architecture, requirements, and key performance indicators for future aerial wireless networks supported by 6G for urban air mobility (UAM). Furthermore, the key enabling technologies and future challenges for incorporating aerial wireless networks in $6 \mathrm{G}$ are reviewed and discussed to support the expected massive deployment of aerial vehicles.
In the third article, entitled "Smarter Grid in the 5G Era: Integrating Power Internet of Things With Cyber Physical System", as the full deployment of the Internet of Things in the power grid (a.k.a. power Internet of Things or PIoT), the newly introduced information flow together with inherent energy flow, makes more efficient power generation, transmission, distribution, and consumption. To further exploit the precious energy and the latest 5G technologies, Liu et al. add a value flow in the smart grid, mainly including the value created by innovative services and market mechanisms and the value added by the information flow. Specifically, this article develops a conceptual framework of the cyber-physical power system (CPPS) by integrating PIoT with cyber-physical systems. The resulting CPPS carries out holistic perception and ubiquitous connection of distributed energy sources and electrical facilities and builds up a smarter power grid with global information interaction, intelligent decision-making, and real-time agile control.

The fourth article, entitled "Numerous Factors Affecting Performance of NOMA for Massive Machine-Type Communications in B5G Systems", by Yan et al. says that with the development of massive machine-type communications (mMTC), services for a huge number of user terminals should be provided simultaneously, so it focuses on the design problems of NOMA scheme and evaluates the impacts of numerous factors, such as channel encoding, channel decoding, repetition number, multiuser detector and the number of receiver antennas, on the performance of NOMA. It is surprised to find that some conclusions drawn from orthogonal multiple-access systems may be invalid for NOMA systems. The factors which have a significant impact on the performance of NOMA should be paid more attention to in the system design. The analysis and evaluation results shine more light on how to design an effective NOMA scheme by considering both transmitter and receiver to fulfill the requirements of mMTC for beyond $5 \mathrm{G}$ systems.

Finally, the last article, entitled "Experimental Demonstration of a mmWave Passive Access Point Extender Based on a Binary Reconfigurable Intelligent Surface", authored by Popov et al. In this article, Popov et al. redirect beams coming from base stations at many locations with reconfigurable intelligent surfaces (RIS) in order to increase their coverage even in cluttered environments. Specifically, a binary tunable metasurface operating at $28 \mathrm{GHz}$, based on standard PCB and off the shelves PIN diodes, is described and experimentally demonstrated. The experimental results show that it can be used as a RIS that beamforms an incoming plane wave at a given angle to one or several outgoing plane waves at angles reconfigurable in real-time. More importantly, the author uses this $20 \mathrm{~cm} \times 20 \mathrm{~cm}$ RIS alongside software-defined radio and up/down converters at $28 \mathrm{GHz}$, and demonstrates a wireless link between an emitter and a receiver $10 \mathrm{~m}$ away, in a non-line of sight configuration, hence proving the validity of the approach.

Our Guest Editor team is pleased with the technical depth and span of this Special Section in Frontiers in Communications and Networks, and also recognizes that it cannot cover all 5G-andbeyond communications issues for smart cities. We sincerely thank all the authors and reviewers for the tremendous efforts and of 
course the Editor-in-Chief and Staff Members for their great guidance. We hope that the readers will enjoy this special section.

\section{AUTHOR CONTRIBUTIONS}

All authors listed have made a substantial, direct, and intellectual contribution to the work and approved it for publication.

Conflict of Interest: The authors declare that the research was conducted in the absence of any commercial or financial relationships that could be construed as a potential conflict of interest.
Publisher's Note: All claims expressed in this article are solely those of the authors and do not necessarily represent those of their affiliated organizations, or those of the publisher, the editors and the reviewers. Any product that may be evaluated in this article, or claim that may be made by its manufacturer, is not guaranteed or endorsed by the publisher.

Copyright () 2022 Xia, Lai, Hu, Liu, Thomos, Liu, Han, Bohara and Sali. This is an open-access article distributed under the terms of the Creative Commons Attribution License (CC BY). The use, distribution or reproduction in other forums is permitted, provided the original author(s) and the copyright owner(s) are credited and that the original publication in this journal is cited, in accordance with accepted academic practice. No use, distribution or reproduction is permitted which does not comply with these terms. 